\title{
Percutaneous Bullectomy in Conjunction with Endobronchial Valve Placement as an Alternative to Surgical Management of Giant Bullae
}

\author{
Aditya Goud ${ }^{a}$ William Krimsky ${ }^{b} \quad$ Marry Caldwel $^{a} \quad$ Brandon Perry $^{a}$ \\ Pooya Heiraty $^{\mathrm{a}}$ Saiyad Sarkar ${ }^{\mathrm{b}}$ Daniel P. Harley ${ }^{\mathrm{c}}$ Stephen Selinger ${ }^{\mathrm{b}}$ \\ Departments of ${ }^{\mathrm{a}}$ Internal Medicine, ${ }^{\mathrm{b}}$ Pulmonary and Critical Care Medicine and ${ }^{\mathrm{c}}$ Thoracic Surgery, \\ MedStar Franklin Square Hospital Center, Baltimore, Md., USA
}

\section{Established Facts}

- Current treatment options for giant bulla involve surgical options of endobronchial valve placement, lobectomy or resection, either via video-assisted thoracoscopic surgery or open technique or Monaldi technique.

\section{Novel Insights}

- In the event of relative/absolute contraindication to the well-known conventional setting, a novel, less invasive procedure that involves the use of an endobronchial valve proximally with transthoracic needle decompression has been described with good short- and long-term outcomes.

\section{Key Words}

Giant bulla Endobronchial valves · Percutaneous bullectomy

\begin{abstract}
We present the first reported case of the treatment and management of a giant bulla using percutaneous bullectomy and endobronchial valve placement. A 74-year-old woman with chronic obstructive pulmonary disease and a known large bulla in the left chest presented to the emergency department with acute-onset confusion after a traumatic fall. She was subsequently diagnosed with an intracranial hemorrhage in the distribution of the right basal ganglia. Chest imaging revealed a giant apical bulla occupying $80 \%$ of the
\end{abstract}

left hemithorax. In addition, there was midline shift away from the affected side associated with volume loss in the right hemithorax and no radiographic evidence of aeration in the remainder of the left lung. Arterial blood gas analysis revealed significant hypercapnia. Surgical bullectomy was not an option, and thus, a novel approach was utilized to treat this patient.

(c) 2016 S. Karger AG, Basel

\section{Case}

A 74-year-old woman with known oxygen-dependent chronic obstructive pulmonary disease-acquired secondary polycythemia vera and a known large bulla that occupied $25 \%$ of the left hemithorax presented to the emergency room with progressive dys-

\section{KARGER}

E-Mail karger@karger.com www.karger.com/res (c) 2016 S. Karger AG, Basel

0025-7931/16/0916-0523\$39.50/0
William Krimsky, MD

Department of Pulmonary and Critical Care Medicine MedStar Franklin Square Hospital Center 9103 Franklin Square Drive, Suite 300, Baltimore, MD 21237 (USA) E-Mail william.krimsky@ medstar.net 


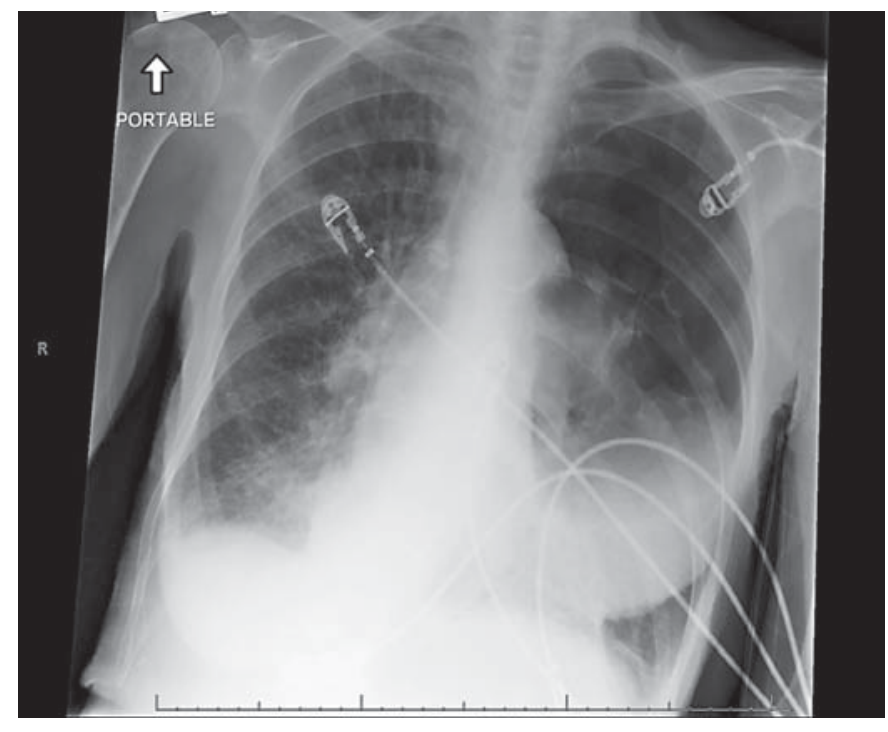

Fig. 1. Initial chest radiograph.

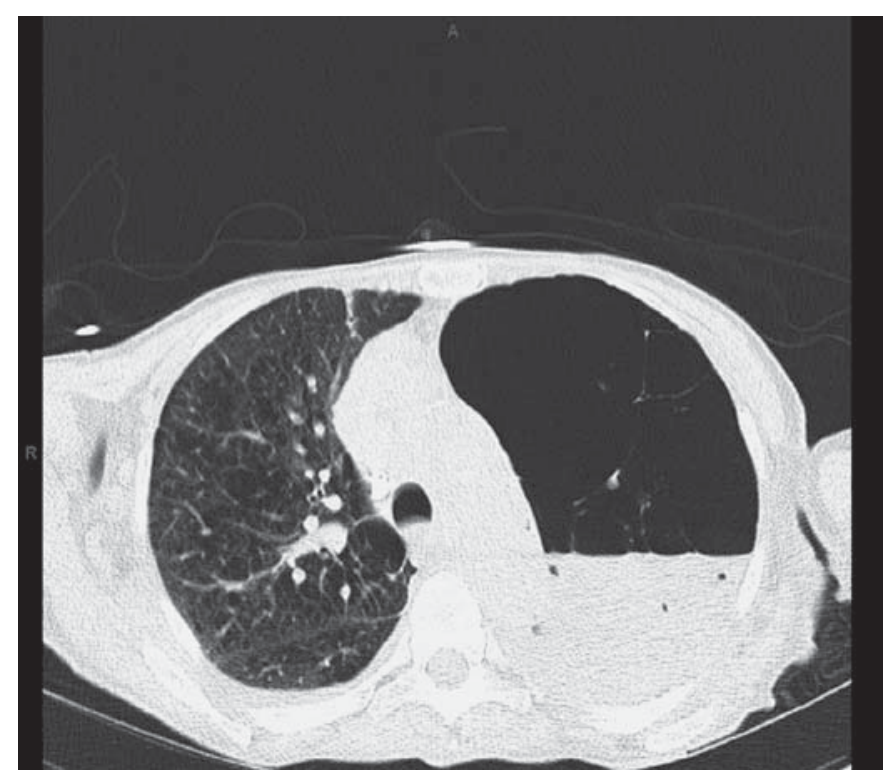

Fig. 2. Chest CT showing a giant bulla occupying $80 \%$ of the left hemithorax.

pnea, confusion and left-sided weakness $12 \mathrm{~h}$ after a mechanical fall. At presentation, her vital signs demonstrated a blood pressure of $100 / 68 \mathrm{~mm} \mathrm{Hg}$, heart rate $85 \mathrm{bpm}$, temperature $36.8^{\circ} \mathrm{C}$, and oxygen saturation of $94 \%$ on 2 liters. Computed tomography (CT) of the head demonstrated an acute intracranial hemorrhage in the distribution of the right basal ganglia measuring $5.0 \times 3.2 \mathrm{~cm}$. The initial chest radiograph revealed the presence of a large bulla now occupying about $80 \%$ of the left hemithorax with tracheal deviation to the right (fig. 1). A chest CT was performed that demonstrated a giant bulla occupying $80 \%$ of the left hemithorax. In addition, there was complete atelectasis of the remainder of the left lung and midline shift away from the affected side associated with volume loss in the right hemithorax. There was no evidence of a pneumothorax (fig. 2).

In the emergency department, arterial blood gas $(\mathrm{ABG})$ analysis demonstrated a $\mathrm{pH}$ of 7.26, $\mathrm{PCO}_{2}>100 \mathrm{~mm} \mathrm{Hg}, \mathrm{PO}_{2} 66 \mathrm{~mm}$ $\mathrm{Hg}$, and bicarbonate $53.1 \mathrm{mEq} / \mathrm{l}$ on $3 \mathrm{l} / \mathrm{min}$ of nasal canula. She was started on noninvasive bilevel positive airway pressure (BiPap) of $14 / 5 \mathrm{~cm} \mathrm{H}_{2} \mathrm{O}$. She became obtunded and a repeat ABG was done demonstrating progressive hypercapnia.

Despite selective intubation of the right main stem bronchus, gas exchange continued to deteriorate with a repeat ABG demonstrating a $\mathrm{pH}$ of 7.10 , with no clinical or radiographic improvement. In addition, there continued to be mediastinal shift away from the affected side. Given the hypercapnia, the acidosis, and the progressive deterioration of her mental status, a minimally invasive approach to avoid further distension of the bulla was considered.

\section{Clinical Discussion}

A giant bulla is defined as a bulla that occupies a minimum of $30 \%$ of a hemithorax [1]. Giant bullae are initially detected on a chest radiograph, and are further evaluated using chest CT scanning to determine associated complications [2]. In this case, the chest CT done in the hospital revealed profound progression of a known large bulla that occupied $80 \%$ of the left hemithorax with midline shift and volume loss on the right. A previous chest CT done 4 months prior to this event demonstrated that the bulla occupied $20 \%$ of the left hemithorax without any midline shift and with good aeration to the remainder of the left lung. Surgical procedures for bullae, such as stapling resection of the bullae with pleurodesis, either via video-assisted thoracoscopic surgery or open technique, versus a modified Monaldi technique [3] or lobectomy, were not an option because of the high risk of surgery $[4,5]$. Invasive surgical options were deferred as a last resort. In this case, placing a closed-tube thoracostomy is contraindicated as it may lead to catastrophic air leaks.

To date, less invasive options for the treatment of giant bulla have largely consisted of strategic placement of endobronchial valves (EBVs) into the affected bronchi subtending the bulla. In the setting of severe bullous disease or in respiratory insufficiency secondary to bullous emphysema, placement of EBVs aids in the deflation of the bullae $[6,7]$ and can improve respiratory function $[8$, 9]. There is also a single case report of transbronchial decompression using needle aspiration of proximal bullae. However, this was done without airway isolation, i.e. without placement of EBVs. There has also been the de- 
scription of intrabullous bronchoscopic delivery of autologous blood in a nonemergent context [10]. In our patient, the location of the bulla, the use of positive pressure ventilation, the risks of a persistent air leak, and the development of persistent bronchopleural or alveolarpleural fistula were considered to be too high to employ transbronchial decompression [11].

A novel approach was considered which was designed to isolate the affected area while allowing for emergent drainage of the bulla such that gas exchange and ventilatory mechanics might be restored. An approach that combined placement of an EBV proximally with transthoracic needle drainage and decompression was attempted in the hope that this would allow for appropriate protection of the affected area while ensuring adequate ventilation of the remaining lung parenchyma.

\section{Intervention}

The patient underwent bronchoscopy through an endotracheal tube. The left bronchus was visualized and the various segmental airways of the left upper lobe were measured. Two 7-mm intrabronchial valves from one supplier (Olympus Medical Co.) were inserted. The first valve was placed in the lingula, and the second in the apicoposterior and anterior segments of the left upper lobe. The details of the valve design, function and insertion technique have previously been described [12]. Images were then reviewed. Given the continued deterioration and the critical nature of the situation, a left closedtube thoracostomy was inserted into the bulla. A 9.0Fr/29-cm Cook medical chest tube was used. A postoperative chest radiograph showed successful reinflation of the remainder of the left lung with improved aeration of the right lung along with resolution of the mediastinal shift (fig. 3). Gas exchange improved dramatically postoperatively and a repeat ABG demonstrated a pH of 7.46, $\mathrm{PCO}_{2} 37.7 \mathrm{~mm} \mathrm{Hg}, \mathrm{PO}_{2} 50.7 \mathrm{~mm} \mathrm{Hg}$, and bicarbonate $40.1 \mathrm{mEq} / \mathrm{l}$. She was extubated the next day as the $\mathrm{pH}$ continued to improve and $\mathrm{PCO}_{2}$ normalized. Her sensorium subsequently cleared and a repeat head CT showed no change in the hemorrhage. The small-bore chest tube was subsequently removed and she was discharged 4 days later. It is now more than 2 years since the initial event and she remains clinically and radiographically stable, without recurrence of the bulla (fig. 4). She continues to be followed clinically by one of the authors.

Less Invasive Technique for the Treatment of Giant Bullae

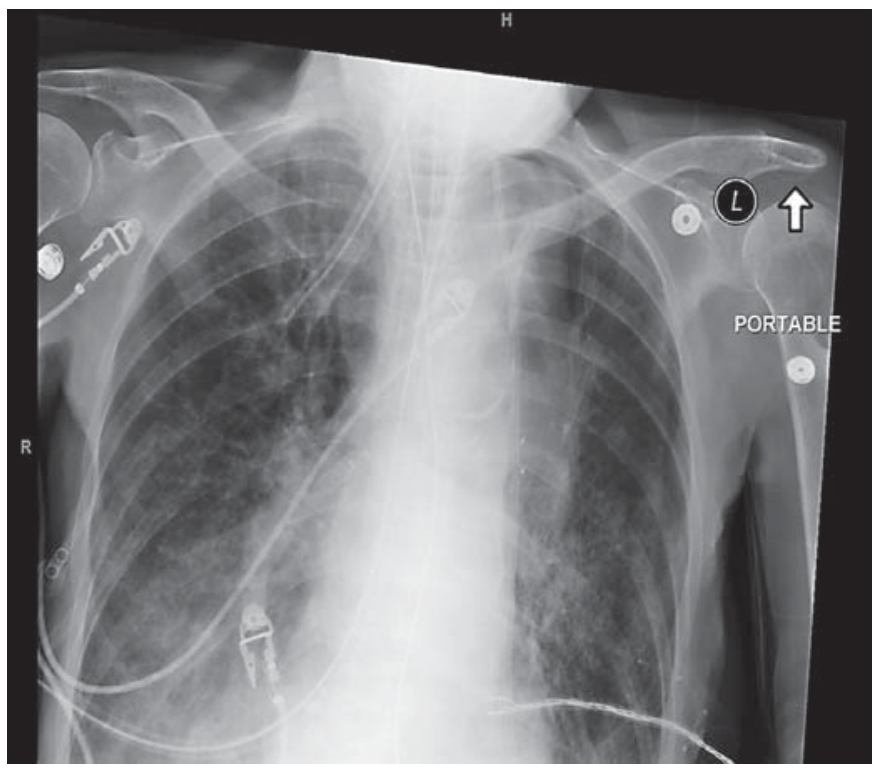

Fig. 3. Postoperative chest radiograph.

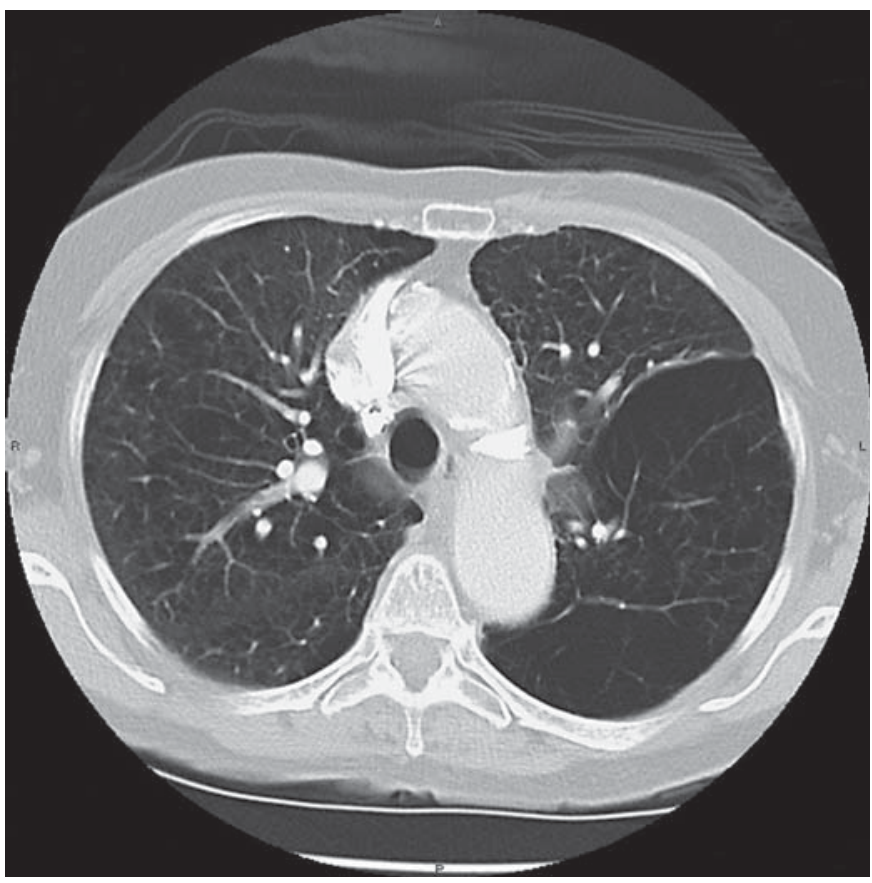

Fig. 4. Chest CT after 2 years showing no recurrence of the bulla.

\section{Conclusion}

The approach to treatment options for a relatively stable patient with giant bullae vary widely from close monitoring to resection via thoracotomy. In our case of an unstable patient who urgently required optimization of gas ex- 
change in the setting of a giant bullae and intracerebral hemorrhage, we describe a less invasive technique that has not been described thus far in the literature. It has now been demonstrated that the benefits of recruiting viable lung parenchyma and reducing the space-occupying effect of a bulla immediately using an EBV in conjunction with drainage and decompression can be both safe and effective. This technique may be superior in preserving the remaining lung parenchyma. Insertion of a drain followed by bronchial isolation to stop the air leak could allow placement of the valve into the precise lung segment involved.

Lung re-expansion was both successful and ultimately lifesaving given the patient's physiologic derangements upon admission. This minimally invasive approach needs to be further studied to determine long-term outcomes, but this technique can certainly offer another approach to a critically ill patient with bullous disease.

\section{Acknowledgements}

We would like to thank the Department of Pulmonary and Critical Care Medicine for their continued support.

\section{Statement of Ethics}

Informed consent was obtained from the patient and his family for educational use of the abovementioned data and no personal patient information has been disclosed.

\section{Financial Disclosure and Conflicts of Interest}

None of the authors have any financial or personal bias that would inappropriately compromise the publication of this work.

\section{References}

1 Thurlbeck WM: Pathophysiology of chronic obstructive pulmonary disease. Clin Chest Med 1990;11:389-403.

2 Sharma N, Justaniah AM, Kanne JP, Gurney JW, Mohammed T-LH: Vanishing lung syndrome (giant bullous emphysema): CT findings in 7 patients and a literature review. J Thorac Imaging 2009;24:227-230.

- 3 Greenberg JA, Singhal S, Kaiser LR: Giant bullous lung disease: evaluation, selection, techniques, and outcomes. Chest Surg Clin N Am 2003;13:631-649.

-4 Ohta M, Nakahara K, Yasumitsu T, Ohsugi T, Maeda M, Kawashima Y: Prediction of postoperative performance status in patients with giant bulla. Chest 1992;101:668-673.

5 Martinez FJ, Chang A: Surgical therapy for chronic obstructive pulmonary disease. Semin Respir Crit Care Med 2005;26:167-191.
6 Noppen M, Tellings J-C, Dekeukeleire T, et al: Successful treatment of a giant emphysematous bulla by bronchoscopic placement of endobronchial valves. Chest 2006;130:15631565.

7 Santini M, Fiorelli A, Vicidomini G, Di Crescenzo VG, Messina G, Laperuta P: Endobronchial treatment of giant emphysematous bullae with one-way valves: a new approach for surgically unfit patients. Eur J Cardiothorac Surg 2011;40:1425-1431.

-8 Tsujino K, Sasada S, Kodama M, Ishihara H, Kawase I: Severe bullous emphysema and hypercapnia successfully treated by bronchoscopic lung volume reduction. Respirology 2009;14:907-909.
9 Sexton P, Garrett JE, Rankin N, Anderson G: Endoscopic lung volume reduction effectively treats acute respiratory failure secondary to bullous emphysema. Respirology 2010;15: 1141-1145.

10 Zoumot Z, Kemp SV, Caneja C, Singh S, Shah PL: Bronchoscopic intrabullous autologous blood instillation: a novel approach for the treatment of giant bullae. Ann Thorac Surg 2013;96:1488-1491.

11 Bhattacharyya P, Sarkar D, Nag S, Ghosh S, Roychoudhury S: Transbronchial decompression of emphysematous bullae: a new therapeutic approach. Eur Respir J 2007;29: 1003-1006.

12 Sterman DH, Mehta AC, Wood DE, et al: A multicenter pilot study of a bronchial valve for the treatment of severe emphysema. Respiration 2010;79:222-233. 